\title{
Improvement of GPS Precise Point Positioning Accuracy in Urban Canyons - Further Results
}

\author{
Masahiro Ozaki, Masaharu Ohashi, Yukihiro Kubo and Sueo Sugimoto \\ Dept. of Electrical and Electronic Engineering, Ritsumeikan University \\ 1-1-1 Noji-Higashi, Kusatsu City, Shiga 525-8577, Japan \\ Tel: +81-(0)77-561-5972, FAX: +81-(0)77-561-2663 \\ E-mail: ykubo@se.ritsumei.ac.jp
}

\begin{abstract}
In this paper, practical methods for improving GPSPPP (Global Positioning System-Precise Point Positioning) accuracy in urban canyons are presented and examined in real severe circumstances. In our previous work, the method for improving positioning accuracy is presented by using a barometric pressure sensor. And the usefulness of the method was shown by the experiments such that the number of satellites used in the position calculation was artificially decreased to 3 . In this paper, the algorithm proposed in our previous work is tested for the automobile which runs in real severe circumstances such as near the skyscrapers, raised expressways and the feasibility and usefulness of the proposed method with few satellites are shown.
\end{abstract}

\section{Introduction}

In this paper, practical methods for improving GPSPPP (Global Positioning System-Precise Point Positioning) accuracy in urban canyons are presented and examined in real severe circumstances. We have already developed PPP algorithms based on GR (Gnss Regression) models [1]-[3]. Our PPP algorithm achieved the positioning accuracy in decimeter level. The algorithm does not require the real time transmitted correction information. Therefore our PPP algorithm can be easily implemented without any external online received data. In [4], Several techniques to improve PPP quality such as using the Doppler measurement, precise modeling of the receiver clock error and the map information have been proposed and successfully integrated into a PPP algorithm which is available in case that there are few (only two or three) visible satellites from automobiles moving on the street in urban canyons. Especially the map information functions efficiently for accuracy improvement, it is used to give a coarse height (altitude) information to keep the continuous positioning and its accuracy when the number of visible satellites decreases to less than four. However, because the height of map information is basically the height of the terrain, it is sometimes incorrect when the vehicle runs on elevated roads, overpass and so on. Therefore, in [5], the method of [4] was improved by utilizing the barometric pres- sure sensor to obtain the vertical variation as an additional information. And the usefulness of the method was shown by the experiments such that the number of satellites used in the position calculation was artificially decreased to 3 . In this paper, the algorithm proposed in [5] is tested for the automobile which runs in real severe circumstances such as near the skyscrapers, raised expressways. In such circumstances, with the standard point positioning method [3], large positioning errors may often occur due to multipath effects and cycle slips, and sometimes we can not obtain position solution because there are no enough satellites. On the other hand, from the conclusions in $[4,5]$, it can be expected that our algorithm can work well in such circumstances.

\section{Measurement Model}

At first, based on [1]-[3], the measurement models for PPP are shown. Though the models shown here are for only GPS(Global Positioning System), the natural extensions for multiple frequencies and GNSS(Global Navigation Satellite Systems) systems can be similarly formulated. We consider the following fundamental measurements of the pseudoranges $\rho_{C A, u}^{p}(t), \rho_{P Y, u}^{p}(t)$ based on $\mathrm{C} / \mathrm{A}$ and $\mathrm{P}(\mathrm{Y})$ codes, and $L_{1}, L_{2}$ bands carrier phases $\varphi_{L 1, u}^{p}(t), \varphi_{L 2, u}^{p}(t)$ as follows:

$$
\begin{aligned}
\rho_{C A, u}^{p}(t)= & r_{u}^{p}\left(t, t-\tau_{u}^{p}\right)+\delta I_{u}^{p}(t)+\delta T_{u}^{p}(t) \\
& +c\left[\delta t_{u}(t)-\delta t^{p}\left(t-\tau_{u}^{p}\right)\right] \\
& +\delta b_{C A, u}-\delta b_{C A}^{p}+e_{C A, u}^{p}(t), \\
\rho_{P Y, u}^{p}(t)= & r_{u}^{p}\left(t, t-\tau_{u}^{p}\right)+\frac{f_{1}^{2}}{f_{2}^{2}} \delta I_{u}^{p}(t)+\delta T_{u}^{p}(t) \\
& +c\left[\delta t_{u}(t)-\delta t^{p}\left(t-\tau_{u}^{p}\right)\right] \\
& +\delta b_{P Y, u}-\delta b_{P Y}^{p}+e_{P Y, u}^{p}(t), \\
\Phi_{L 1, u}^{p}(t)= & \lambda_{1} \varphi_{L 1, u}^{p}(t) \\
= & r_{u}^{p}\left(t, t-\tau_{u}^{p}\right)-\delta I_{u}^{p}(t)+\delta T_{u}^{p}(t) \\
& +c\left[\delta t_{u}(t)-\delta t^{p}\left(t-\tau_{u}^{p}\right)\right] \\
& +\delta b_{L 1, u}-\delta b_{L 1}^{p} \\
& +\lambda_{1} N_{u}^{p}+\lambda_{1} \varepsilon_{u}^{p}(t),
\end{aligned}
$$




$$
\begin{aligned}
\Phi_{L 2, u}^{p}(t)= & \lambda_{2} \varphi_{L 2, u}^{p}(t) \\
= & r_{u}^{p}\left(t, t-\tau_{u}^{p}\right)-\frac{f_{1}^{2}}{f_{2}^{2}} \delta I_{u}^{p}(t)+\delta T_{u}^{p}(t) \\
& +c\left[\delta t_{u}(t)-\delta t^{p}\left(t-\tau_{u}^{p}\right)\right] \\
& +\delta b_{L 2, u}-\delta b_{L 2}^{p} \\
& +\lambda_{2} N_{u}^{p}+\lambda_{2} \varepsilon_{u}^{p}(t)
\end{aligned}
$$

where $c$ is the speed of light $\left(2.99792458 \times 10^{8}[\mathrm{~m} / \mathrm{s}]\right)$, and $f_{*}$ and $\lambda_{*}$ are the central frequency and the wave length of the $L_{*}$ carrier wave $\left(f_{1}=1575.42[\mathrm{MHz}]\right.$, $\left.f_{2}=1227.60[\mathrm{MHz}]\right)$. In (1)-(4), the terms $\left\{\delta b_{C A, u}\right.$, $\left.\delta b_{P Y, u}, \delta b_{L 1, u}, \delta b_{L 2, u}\right\}$ and $\left\{\delta b_{C A}^{p}, \delta b_{P Y}^{p}, \delta b_{L 1}^{p}, \delta b_{L 2}^{p}\right\}$ are the so-called receiver and satellite biases respectively, and in this paper they are ignored. Also $r_{u}^{p}\left(t, t-\tau_{u}^{p}\right)$ is the geometric distance between the receiver $u$ at the time $t$ and the satellite $p$ at the time $t-\tau_{u}^{p}\left(\tau_{u}^{p}\right.$ denotes the travel time from the satellite $p\left(p=1, \ldots, n_{s}\right)$ to the receiver $u$ ). Namely,

$$
\begin{aligned}
r_{u}^{p}(t) \equiv & r_{u}^{p}\left(t, t-\tau_{u}^{p}\right) \\
= & {\left[\left(x_{u}(t)-x^{p}\left(t-\tau_{u}^{p}\right)\right)^{2}+\left(y_{u}(t)-y^{p}\left(t-\tau_{u}^{p}\right)\right)^{2}\right.} \\
& \left.+\left(z_{u}(t)-z^{p}\left(t-\tau_{u}^{p}\right)\right)^{2}\right]^{1 / 2} \\
= & \left\|u(t)-s^{p}\left(t-\tau_{u}^{p}\right)\right\|,
\end{aligned}
$$

where $u \equiv\left[x_{u}, y_{u}, z_{u}\right]^{\mathrm{T}}$ and $s^{p} \equiv\left[x^{p}, y^{p}, z^{p}\right]^{\mathrm{T}}$ are the user (unknown) and satellite positions, respectively. Also $n_{s}$ shows the number of the observable satellites. In (1)-(4), $\delta I_{u}^{p}$ and $\delta T_{u}^{p}$ are the delay or the advance associated with the transmission of the $L_{1}$ signal through the ionosphere and the troposphere, respectively. $\delta t_{u}$ and $\delta t^{p}$ are the clock errors of the receiver $u$ and the satellite $p . N_{u}^{p}$ denotes integer ambiguity between the satellite $p$ and the receiver $u$, and $\varepsilon^{p}$, $e^{p}$ denote measurement errors. Because the measurement models (1)(4) are nonlinear due to the geometric distance term $r_{u}^{p}$, it is linearized by the first order Taylor series approximation around the initial estimate $\hat{u}$ and $\hat{s}^{p}$ as follows:

$$
\begin{aligned}
r_{u}^{p} & \cong r_{\hat{u}}^{\hat{p}}+g_{\hat{u}}^{\hat{p}}\left[u-s^{p}-\left(\hat{u}-\hat{s}^{p}\right)\right] \\
& =\left\|\hat{u}-\hat{s}^{p}\right\|+\frac{\left(\hat{u}-\hat{s}^{p}\right)^{\mathrm{T}}}{\left\|\hat{u}-\hat{s}^{p}\right\|}\left[u-s^{p}-\left(\hat{u}-\hat{s}^{p}\right)\right] \\
& =\frac{\left(\hat{u}-\hat{s}^{p}\right)^{\mathrm{T}}}{\left\|\hat{u}-\hat{s}^{p}\right\|}\left(u-s^{p}\right)
\end{aligned}
$$

for $p=1,2, \ldots, n_{s}$, where

$$
g_{\hat{u}}^{\hat{p}} \equiv\left[\frac{\partial r_{u}^{p}}{\partial u}\right]_{u=\hat{u}, s^{p}=\hat{s}^{p}}^{\mathrm{T}}=\frac{\left(\hat{u}-\hat{s}^{p}\right)^{\mathrm{T}}}{\left\|\hat{u}-\hat{s}^{p}\right\|} .
$$

Also the estimate of the satellite position $\hat{s}^{p}$ can be obtained from the broadcast ephemeris or precise orbit information. From (1)-(4), therefore, we have the approximations:

$$
\begin{aligned}
\rho_{C A, u}^{p} \cong & g_{\hat{u}}^{\hat{p}}\left(u-s^{p}\right) \\
& +\delta I_{u}^{p}+\delta T_{u}^{p}+c\left(\delta t_{u}-\delta t^{p}\right) \\
& +\delta b_{C A, u}-\delta b_{C A}^{p}+e_{C A, u}^{p}, \\
\rho_{P Y, u}^{p} \cong & g_{\hat{u}}^{\hat{p}}\left(u-s^{p}\right) \\
& +\frac{f_{1}^{2}}{f_{2}^{2}} \delta I_{u}^{p}(t)+\delta T_{u}^{p}+c\left(\delta t_{u}-\delta t^{p}\right) \\
& +\delta b_{P Y, u}-\delta b_{P Y}^{p}+e_{P Y, u}^{p}, \\
\cong & g_{\hat{u}}^{\hat{p}}\left(u-s^{p}\right) \\
& -\delta I_{u}^{p}+\delta T_{u}^{p}+c\left(\delta t_{u}-\delta t^{p}\right) \\
& +\delta b_{L 1, u}-\delta b_{L 1}^{p}+\lambda_{1} N_{L 1, k}^{p}+\lambda_{1} \varepsilon_{L 1, u}^{p}, \\
\Phi_{L 1, u}^{p}, & g_{\hat{u}}^{\hat{p}}\left(u-s^{p}\right) \\
\Phi_{L 2, u}^{p} & -\frac{f_{1}^{2}}{f_{2}^{2}} \delta I_{u}^{p}+\delta T_{u}^{p}+c\left(\delta t_{u}-\delta t^{p}\right) \\
& +\delta b_{L 2, u}-\delta b_{L 2}^{p}+\lambda_{2} N_{L 2, k}^{p}+\lambda_{2} \varepsilon_{L 2, u}^{p} .
\end{aligned}
$$

By differentiation of (10) and (11), we have approximately the Doppler shift data $D_{L 1, u}^{p}$ and $D_{L 2, u}^{p}$ for $L 1$ and $L 2$ carrier frequencies, respectively, as follows:

$$
\begin{aligned}
& \lambda_{1} D_{L 1, u}^{p} \cong g_{\hat{u}}^{\hat{p}}\left(\dot{u}-\dot{s}^{p}\right)+c \dot{\delta} t_{u}+b_{D L 1}+\lambda_{1} \varepsilon_{D L 1, u}^{p}, \\
& \lambda_{2} D_{L 2, u}^{p} \cong g_{\hat{u}}^{\hat{p}}\left(\dot{u}-\dot{s}^{p}\right)+c \dot{\delta} t_{u}+b_{D L 2}+\lambda_{2} \varepsilon_{D L 2, u}^{p},
\end{aligned}
$$

where $c \dot{\delta} t_{u}$ is receiver clock drift rate, $b_{D L *}$ are biased constants, $\varepsilon_{D L *, u}^{p}$ are measurement noises. Then from (8)-(13), we have the following vector regression equation:

$$
y_{u}^{s}=H_{\hat{u}}^{\hat{s}} \theta_{u}+v_{u},
$$

where

$$
y_{u}^{s} \equiv\left[\begin{array}{c}
\rho_{C A, u}^{s} \\
\rho_{P Y, u}^{s} \\
\Phi_{L 1, u}^{s} \\
\Phi_{L 2, u}^{s} \\
\lambda_{1} D_{L 1, u}^{s} \\
\lambda_{2} D_{L 2, u}^{s}
\end{array}\right], \quad \theta_{u} \equiv\left[\begin{array}{c}
u \\
\dot{u} \\
c \delta t_{u} \\
c \dot{\delta} t_{u} \\
c \delta t^{s} \\
s \\
\dot{s} \\
\delta I_{u} \\
\delta T_{u} \\
\lambda_{1} N_{L 1, u} \\
\lambda_{2} N_{L 2, u} \\
b_{D L 1} \\
b_{D L 2}
\end{array}\right]
$$

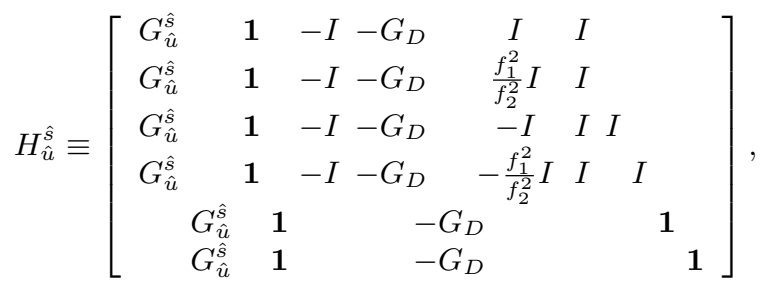


$v_{u} \equiv\left[e_{C A, u}^{\mathrm{T}}, e_{P Y, u}^{\mathrm{T}}, \lambda_{1} \varepsilon_{L 1, u}^{\mathrm{T}}, \lambda_{2} \varepsilon_{L 2, u}^{\mathrm{T}}, \lambda_{1} \varepsilon_{D L 1, u}^{\mathrm{T}}, \lambda_{2} \varepsilon_{D L 2, u}^{\mathrm{T}}\right]^{\mathrm{T}}$,

where $I$ denote the $n_{s} \times n_{s}$ unit matrix and $\mathbf{1} \equiv$ $[1,1, \cdots, 1]^{\mathrm{T}}: \quad n_{s} \times 1$ vector. Also define the $n_{s} \times 3$ matrix:

$$
G_{\hat{u}}^{\hat{s}} \equiv\left[\begin{array}{c}
g_{\hat{\hat{1}}}^{\hat{1}} \\
g_{\hat{u}}^{2} \\
\vdots \\
g_{\hat{\hat{u}}}^{\hat{\hat{n}_{s}}}
\end{array}\right]
$$

Furthermore, we define a block diagonal matrix with the size $\left(n_{s} \times 3 n_{s}\right)$ :

$$
G_{D, \hat{u}}^{\hat{s}} \equiv\left[\begin{array}{ccccc}
g_{\hat{u}}^{\hat{1}} & O & O & \cdots & O \\
O & g_{\hat{u}}^{\hat{2}} & O & \cdots & O \\
\vdots & & \ddots & & \vdots \\
\vdots & & & \ddots & O \\
O & \cdots & \cdots & O & g_{\hat{u}}^{\hat{n}_{s}}
\end{array}\right]
$$

Now we assume that the information of the satellite position $s$, the time derivative of the satellite position $\dot{s}$, the satellite clock error $c \delta t^{s}$ as well as the delay or the advance due to the ionospheric and tropospheric effects, $\delta I_{u}^{\mathrm{T}}$ and $\delta T_{u}^{\mathrm{T}}$ are provided with errors as follows

$$
\begin{aligned}
& \hat{s}=\left[\begin{array}{llllllllllll}
\mathbf{0}_{3} & \mathbf{0}_{3} & \mathbf{0} & \mathbf{0} & \mathrm{I}_{3 n_{s}} O & O & O & O & O & O & \mathbf{0} & \mathbf{0}
\end{array}\right] \theta_{u}+e_{s},(20) \\
& \hat{\dot{s}}=\left[\begin{array}{lllllllllllll}
\mathbf{0}_{\mathbf{3}} & \mathbf{0}_{\mathbf{3}} & \mathbf{0} & \mathbf{0} & O & I_{3 n_{s}} O & O & O & O & O & \mathbf{0} & \mathbf{0}
\end{array}\right] \theta_{u}+e_{\dot{s}},
\end{aligned}
$$

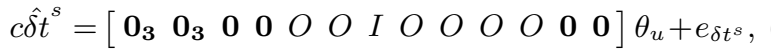

$$
\begin{aligned}
& \hat{\delta I_{u}}=\left[\begin{array}{lllllllllllll}
\mathbf{0}_{\mathbf{3}} & \mathbf{0}_{\mathbf{3}} & \mathbf{0} & \mathbf{0} & O & O & O & I & O & O & O & \mathbf{0} & \mathbf{0}
\end{array}\right] \theta_{u}+e_{\delta I_{u}}, \\
& \delta T_{u}=\left[\begin{array}{lllllllllllll}
\mathbf{0}_{\mathbf{3}} & \mathbf{0}_{\mathbf{3}} & \mathbf{0} & \mathbf{0} & O & O & O & O & O & \text { O O O } & \mathbf{0} & \mathbf{0}
\end{array}\right] \boldsymbol{\theta}_{u}+e_{\delta T_{u}},
\end{aligned}
$$

where $\mathbf{0}_{3}$ is a $n_{s} \times 3$ zero matrix, and the errors $e_{s}, \cdots, e_{\delta T_{u}}$ are assumed to be Gaussian white noise processes. From (20)-(24), we have

$$
\begin{aligned}
s & =\hat{s}-e_{s}, \\
\dot{s} & =\hat{\dot{s}}-e_{\dot{s}} \\
c \delta t^{p} & =c \hat{\delta} t^{s}-e_{\delta t^{s}}, \\
\delta I_{u} & =\hat{\delta I_{u}}-e_{\delta I_{u}}, \\
\delta T_{u} & =\delta \hat{T}_{u}-e_{\delta T_{u}} .
\end{aligned}
$$

Substituting the above equations (25)-(29) into the GR equation (14), we have are derived as follows.

$$
y_{u} \equiv\left[\begin{array}{c}
y_{C A, \hat{u}}^{\hat{s}} \\
y_{P Y, \hat{u}}^{\hat{s}} \\
y_{L 1, \hat{u}}^{\hat{s}} \\
y_{L 2, \hat{u}}^{\hat{s}} \\
y_{D L 1, \hat{u}}^{\hat{s}} \\
y_{D L 2, \hat{u}}^{\hat{s}}
\end{array}\right]=C_{\hat{u}}^{\hat{s}}\left[\begin{array}{c}
u \\
\dot{u} \\
c \delta t_{u} \\
c \dot{\delta} t_{u} \\
\lambda_{1} N_{L 1, u} \\
\lambda_{2} N_{L 2, u} \\
b_{D L 1} \\
b_{D L 2}
\end{array}\right]+v
$$

where

$$
\begin{aligned}
& y_{C A, \hat{u}}^{\hat{s}} \equiv \rho_{C A, u}^{s}+G_{D, \hat{u}}^{\hat{s}} \hat{s} \\
& +c \hat{\delta} t^{s}-\hat{\delta I_{u}}-\hat{\delta T_{u}}, \\
& y_{P Y, \hat{u}}^{\hat{s}} \equiv \rho_{P Y, u}^{s}+G_{D, \hat{u}}^{\hat{s}} \hat{s} \\
& +c \hat{\delta} t^{s}-\frac{f_{1}^{2}}{f_{2}^{2}} \hat{\delta I_{u}}-\hat{\delta T_{u}}, \\
& y_{L 1, \hat{u}}^{\hat{s}} \equiv \Phi_{L 1, u}^{s}+G_{D, \hat{u}}^{\hat{s}} \hat{s} \\
& +c \hat{\delta} t^{s}+\hat{\delta} I_{u}-\hat{\delta T} \hat{T}_{u}, \\
& y_{L 2, \hat{u}}^{\hat{s}} \equiv \Phi_{L 2, u}^{s}+G_{D, \hat{u}}^{\hat{s}} \hat{s} \\
& +c \hat{\delta \delta} t^{s}+\frac{f_{1}^{2}}{f_{2}^{2}} \hat{\delta I} I_{u}-\hat{\delta T_{u}}, \\
& y_{D L 1, \hat{u}}^{\hat{s}} \equiv \lambda_{1} D_{L 1, u}^{s}+G_{D, \hat{u}}^{\hat{s}} \hat{\dot{s}} \text {, } \\
& y_{D L 2, \hat{u}}^{\hat{s}} \equiv \lambda_{2} D_{L 2, u}^{s}+G_{D, \hat{u}}^{\hat{s}} \hat{\dot{s}} \text {, } \\
& C_{\hat{u}}^{\hat{s}}=\left[\begin{array}{rrrrrrrr}
G_{\hat{u}}^{\hat{s}} & & \mathbf{1} & & & & & \\
G_{\hat{\hat{s}}}^{\hat{s}} & & \mathbf{1} & & & & & \\
G_{\hat{\hat{s}}}^{\hat{s}} & & \mathbf{1} & & I & & & \\
G_{\hat{u}}^{\hat{s}} & & \mathbf{1} & & & I & & \\
& G_{\hat{\hat{u}}}^{\hat{s}} & & \mathbf{1} & & & \mathbf{1} & \\
& G_{\hat{u}}^{\hat{s}} & & \mathbf{1} & & & & \mathbf{1}
\end{array}\right],
\end{aligned}
$$

and

$$
v_{t}=\left[\begin{array}{c}
G_{D, \hat{u}}^{\hat{s}} e_{s}+e_{\delta t^{s}}-e_{\delta I_{u}}-e_{\delta T_{u}}+e_{C A, u} \\
G_{D, \hat{u}}^{\hat{s}} e_{s}+e_{\delta t^{s}}-\frac{f_{1}^{2}}{f_{2}^{2}} e_{\delta I_{u}}-e_{\delta T_{u}}+e_{P Y, u} \\
G_{D, \hat{u}}^{\hat{s}} e_{s}+e_{\delta t^{s}}+e_{\delta I_{u}}-e_{\delta T_{u}}+\lambda_{1} \varepsilon_{L 1, u} \\
G_{D, \hat{u}}^{\hat{s}} e_{s}+e_{\delta t^{s}}+\frac{f_{1}^{2}}{f_{2}^{2}} e_{\delta I_{u}}-e_{\delta T_{u}}+\lambda_{2} \varepsilon_{L 2, u} \\
G_{D, \hat{u}}^{\hat{s}} e_{\dot{s}}+\lambda_{1} \varepsilon_{D L 1, u}^{p} \\
G_{D, \hat{u}}^{\hat{s}} e_{\dot{s}}+\lambda_{2} \varepsilon_{D L 2, u}^{p}
\end{array}\right] .
$$

\section{Kalman Filter Formulation}

In this section, the Kalman filtering algorithm for PPP positioning is derived. For this purpose, we derive the so-called state equation as follows.

\subsection{State Equations}

Let us obtain the state equation for positioning. Since the kinematic case requires mathematical models of the moving objects, we often apply one of the dynamical models which are assumed as first-order Markov processes of, the velocity of $u$ (user position), of the acceleration of $u$ (the so-called Singer's moving model [6]), or of the jerk of $u,[7]$, with or without the constraints $[8,9]$. For the land vehicle such as automobiles, we adopt Singer's model for the east-west (E) and northsouth $(\mathrm{N})$ coordinates and a first order Markov model for the velocity of the up-down (U) coordinate frame (see Fig. 1) [3]. Namely, the state vector $\eta$ is defined as follows: 


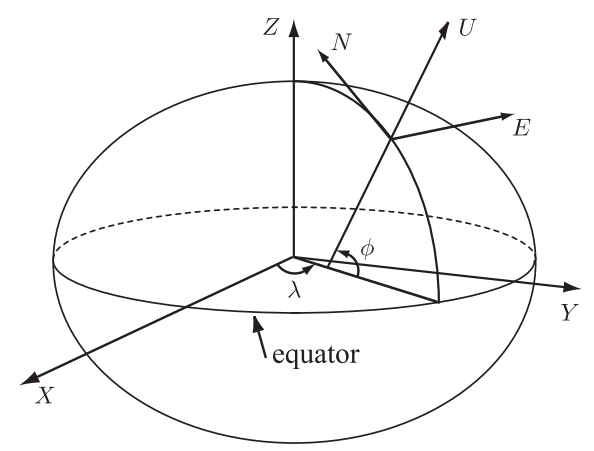

Fig. 1: ECEF-system and ENU-system

$$
\begin{aligned}
\eta_{L} \equiv & {\left[u_{L}^{\mathrm{T}}, \dot{u}_{L}^{\mathrm{T}}, a_{L}^{\mathrm{T}}, c \delta t_{u},\right.} \\
& \left.c \dot{\delta} t_{u}, \lambda_{1} N_{L 1, u}^{\mathrm{T}}, \lambda_{2} N_{L 2, u}^{\mathrm{T}}, b_{D L 1}, b_{D L 2}\right]^{\mathrm{T}} \\
\equiv & {\left[x_{L}, y_{L}, z_{L}, v_{x_{L}}, v_{y_{L}}, v_{z_{L}}, a_{x_{L}}, a_{y_{L}}, c \delta t_{u},\right.} \\
& \left.c \dot{\delta} t_{u}, \lambda_{1} N_{L 1, u}^{\mathrm{T}}, \lambda_{2} N_{L 2, u}^{\mathrm{T}}, b_{D L 1}, b_{D L 2}\right]^{\mathrm{T}},
\end{aligned}
$$

where the subscript $L$ stands for the coordinates in the local frame (or the local-level system, or east-north-up (ENU) system) [3] and we define the user position in the local frame as $u_{L} \equiv\left[x_{L}, y_{L}, z_{L}\right]^{\mathrm{T}} . N_{L 1, u}$ and $N_{L 2, u}$ are $n_{s} \times 1$ ambiguity vectors respectively, where $n_{s}$ is the number of visible satellites. Then the transformation matrix from WGS-84 coordinates to ENU coordinates is given by

$$
T_{W}^{L}=\left[\begin{array}{ccc}
-\sin \lambda & \cos \lambda & 0 \\
-\sin \phi \cos \lambda & -\sin \phi \sin \lambda & \cos \phi \\
\cos \phi \cos \lambda & \cos \phi \sin \lambda & \sin \phi
\end{array}\right]
$$

where $\lambda$ and $\phi$ denote the longitude and latitude, respectively. Let $u_{0}$ in the WGS- 84 coordinate system be an origin in the ENU coordinate system, then any coordinate $u$ in the WGS- 84 system can be described by the ENU system by the relation:

$$
u_{L}=T_{W}^{L}\left(u-u_{0}\right) .
$$

Then by the assumption of the Singer's model, namely the accelerations; $a_{x, L}, a_{y, L}$ of $x_{L}, y_{L}$, respectively, are assumed as the first order Markov processes:

$$
\begin{aligned}
& \dot{a}_{x_{L}}(t)=-\alpha_{x} a_{x_{L}}(t)+w_{a_{x}}(t), \\
& \dot{a}_{y_{L}}(t)=-\alpha_{y} a_{y_{L}}(t)+w_{a_{y}}(t) .
\end{aligned}
$$

Also the velocity of $v_{z, L}$ of $z_{L}$ is assumed as a first order Markov process:

$$
\dot{v}_{z_{L}}(t)=-\alpha_{z} v_{z_{L}}(t)+w_{v_{z}}(t) .
$$

On the other hand, the receiver's clock errors are generally modeled as follows $[10,11]$,

$$
\begin{aligned}
{\left[\begin{array}{c}
c \delta t_{u, t+1} \\
c \dot{\delta} t_{u, t+1}
\end{array}\right] } & =\left[\begin{array}{cc}
1 & \Delta_{t} \\
0 & 1
\end{array}\right]\left[\begin{array}{l}
c \delta t_{u, t} \\
c \dot{\delta} t_{u, t}
\end{array}\right]+\left[\begin{array}{l}
w_{c \delta t_{u}, t} \\
w_{c \dot{\delta} t_{u}, t}
\end{array}\right] \\
& \equiv F_{c \delta t}\left[\begin{array}{c}
c \delta t_{u, t} \\
c \dot{\delta} t_{u, t}
\end{array}\right]+\left[\begin{array}{l}
w_{c \delta t_{u}, t} \\
w_{c \dot{\delta} t_{u}, t}
\end{array}\right]
\end{aligned}
$$

where $\Delta_{t}$ denotes the sampling interval of the receiver's clock error, and the noise $w_{c \delta t_{u}, t}$ and $w_{c \dot{\delta} t_{u}, t}$ are assumed as white Gaussian processes with zero means and variances $q_{c \delta t}$ and $q_{c \dot{\delta} t}$, respectively. Then, we have the following discrete-time state equation:

$$
\eta_{L, t+1}=A_{t} \eta_{L, t}+w_{t}
$$

where

$$
A_{t}=\left[\begin{array}{ccccccc}
A_{11} & & & & & \\
& F_{c \delta t} & & & & \\
& & I & & & \\
& & & I & & \\
& & & & 1 & \\
& & & & & 1
\end{array}\right]
$$

and the $8 \times 8$ matrix $A_{11}$ is given in [3] and the $2 \times 2$ matrix $F_{c \delta t}$ is defined in (45). $w_{t}$ is the Gaussian white noise process with zero mean and covariance matrix $Q_{t}$.

\subsection{Measurement Equations}

Now we assume that $n_{s}$ satellites are observable. Then the measurements (1)-(4), (12) and (13) are obtained from multiple satellites $p=1, \ldots, n_{s}$. Therefore, with the approximation (6), the information of (20)(24) and the relation between the coordinate systems (41), the measurement equation can be expressed by the following vector-matrix form:

$$
y_{L, t} \equiv C_{t} \eta_{L, t}+v_{t}
$$

where $y_{L, t}$ is the $12 n_{s} \times 1$ measurement vector computed from $\mathrm{C} / \mathrm{A}, \mathrm{P}(\mathrm{Y})$ code pseudoranges, $L_{1}, L_{2}$ carrier phases and Doppler measurements. And $C_{t}$ is $12 n_{s} \times\left(2 n_{s}+12\right)$ known matrix such that

$$
C_{t}=\left[\begin{array}{ccccccccc}
G_{L, \hat{u}}^{\hat{s}} & & \mathbf{0}_{2} & \mathbf{1} & & & & & \\
G_{L, \hat{u}}^{\hat{s}} & & \mathbf{0}_{2} & \mathbf{1} & & & & & \\
G_{L, \hat{u}}^{\hat{s}} & \mathbf{0}_{2} & \mathbf{1} & & & I & & & \\
G_{L, \hat{u}}^{\hat{s}} & \mathbf{0}_{2} & \mathbf{1} & & & I & & \\
G_{L, \hat{u}}^{\hat{s}} & \mathbf{0}_{2} & & \mathbf{1} & & & \mathbf{1} & \\
G_{L, \hat{u}}^{\hat{s}} & \mathbf{0}_{2} & & \mathbf{1} & & & & \mathbf{1}
\end{array}\right],
$$

where $\mathbf{0}_{2}$ is a $n_{s} \times 2$ zero matrix, and

$$
G_{L, \hat{u}}^{\hat{s}} \equiv G_{\hat{u}}^{\hat{s}}\left(T_{W}^{L}\right)^{T} .
$$

\section{Additional Measurement from Barometric Pressure Sensor}

By applying the Kalman filter to equations (46) and (48), the position estimation can be implemented. However, in urban canyons, the number of visible satellites often decreases to less than four due to obstacles, multi-path, etc. Then the positioning accuracy may be greatly degraded or the Kalman filter may diverge. In [4], the map information is used to give a coarse height (altitude) information to keep the continuous positioning and its accuracy when the number of visible 
satellites decreases to less than four. The coarse altitude obtained from the map information is added to the measurement equation (48) based on the assumption that the automobiles run on almost flat streets. However, this assumption is not sometimes appropriate because the altitude of the automobile which runs in urban canyons may frequently change due to overpasses, raised expressways and so on. Therefore, the method of [4] is improved to utilize a relatively fine altitude information by using a barometric pressure sensor. The digital barometric pressure sensor utilized in this research is BMP085 of BOSCH which is low cost (about $\$ 5$ ) and can provide height information with high resolution $(0.03[\mathrm{hPa}]$ equivalent to about $0.25[\mathrm{~m}])$. In order to convert an atmospheric pressure value to altitude, the standard atmosphere model [12] can be applied such that

$$
h_{t}=\frac{\left[\left(T_{0}+273.15\right)\left(\frac{P_{t}}{P_{0}}\right)^{-\frac{R L}{g_{o} M}}-\left(T_{0}+273.15\right)\right]}{L},
$$

where $h_{t}$ is altitude of observation point [m] at time $t$, $P_{t}$ is observed barometric pressure $[\mathrm{hPa}], P_{0}$ is barometric pressure of sea level altitude, $g_{o}\left(9.80665\left[\mathrm{~m} / \mathrm{s}^{2}\right]\right)$ is earth's gravity, $T_{0}$ is air temperature of sea level altitude $\left[{ }^{\circ} \mathrm{C}\right], L\left(-0.0065\left[{ }^{\circ} \mathrm{C} / \mathrm{m}\right]\right)$ is temperature lapse rate, $M(0.0289644[\mathrm{~kg} / \mathrm{mol}])$ is atmospheric molar mass, $R(8.31432[\mathrm{~J} / \mathrm{K} / \mathrm{mol}])$ is gas constant [12]. Generally, the model of (51) should be properly calibrated with $P_{0}$ and $T_{0}$ to obtain the altitude above sea level . Therefore, to avoid the calibration problem, the barometric pressure sensor is utilized to provide only the vertical position variation between the successive measurement epochs by using standard pressure of $P_{0}=1013.25$ and temperature $T_{0}=15$. The vertical variation information can be regarded as the additional vertical velocity measurement in Kalman filter as follows.

$$
y_{b, t}=\left(h_{t}-h_{t-1}\right) / \Delta_{t}=v_{z_{L}, t}+v_{b, t},
$$

where $v_{b, t}$ is the measurement noise, $v_{z_{L}, t}$ is the vertical velocity which is the sixth component of the state vector $\eta_{L, t}$ and $\Delta_{t}$ is the interval of the measurement. Therefore, the measurement equation is extended as follows.

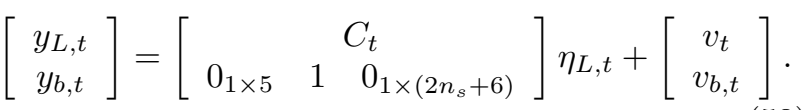

In this paper, the Kalman filter is applied to the state and measurement equations (46) and (53).

\section{Experimental Results}

The experiment of the proposed algorithms has been carried out. In the experiment, the automobile ran in Otsu City area, Shiga, Japan, and the GPS data were obtained by the receiver (NovAtel OEM5) with the antenna (NovAtel GPS-600) on October 11, 2014, from
03:10'20 to $03: 15$ '02 GPST at $1[\mathrm{~Hz}]$ rate $\left(\Delta_{t}=1[\mathrm{~s}]\right)$. The barometric pressure recording system was built by using the pressure sensor BMP085 of BOSCH, u-blox 6 compatible GPS module E-1612-UB and the microcontroller board (Arduino). The GPS module E-1612-UB was used to add the time information to the pressure data. Throughout the experiment, the GPS data at the reference station (Otsu1) of GNSS Earth Observation Network System (GEONET) is also obtained from the Geospatial Information Authority of Japan (GSI). And results of the baseline analysis, i.e. the dual frequency carrier phase differential GPS method, were used as the reference trajectory of the automobile for the evaluation of the experimental results. The averaged baseline length was about $4[\mathrm{~km}]$. Fig. 2 shows the RTK (Real Time Kinematic) results of the baseline analysis. And positioning in East, North and Up directions and the number of satellites used in position calculation. With applying the elevation mask angle of 20 degrees, up to 8 satellites were available during the experiment. In three figures from the top of Fig. 2, the green lines show the results of the baseline analysis.
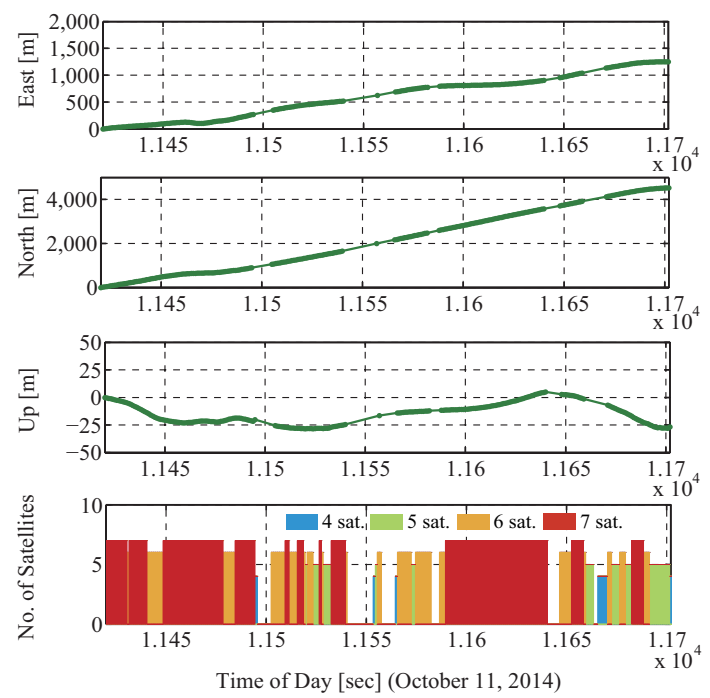

Fig. 2: RTK Results (regarded as the reference trajectory for evaluating PPP results); positions in east, north and up directions, and number of satellites utilized for position calculation

Fig. 3 shows the PPP results; positioning errors in East, North and Up directions and the number of satellites used in position calculation. With applying the elevation mask angle of 20 degrees, up to 7 satellites were available during the experiment. In the experiment, the number of satellites used in the position calculation was artificially decreased to 2 during two periods: 1143011490 and 11678-11702 [sec]. These periods are the entrance and exit of a raised expressway. If the number of satellites was less than 3, the Kalman filter implementation was interrupted and re-initialized as three or more satellites became available. In three figures from the top of Fig. 3, the blue lines show the results of PPP without the barometric pressure data, and the 
red lines show the results of PPP with the barometric pressure data. And Table 1 shows the RMS errors in East, North and Up directions PPP results.
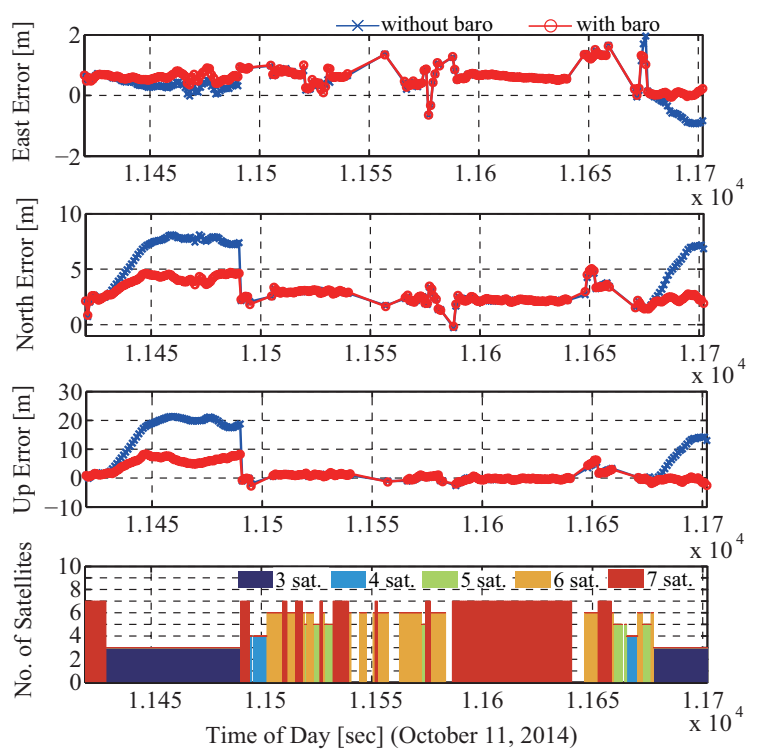

Fig. 3: PPP Results; position errors in east, north and up directions, and number of satellites utilized for position calculation

Table 1: PPP Results; RMS Errors [m]

\begin{tabular}{|c|c|c|}
\hline & without baro & with baro \\
\hline East Error & 0.666 & 0.616 \\
\hline North Error & 4.531 & 3.069 \\
\hline Up Error & 9.777 & 3.451 \\
\hline
\end{tabular}

$*$ baro $=$ barometric pressure

From Fig. 3, Table 1 it can be seen that the Kalman filter performance without the barometric pressure data is quite degraded when the number of satellites is three. In contrast, the accurate positioning can be continuously obtained by using barometric pressure data (proposed method).

\section{Conclusions}

In this paper, the algorithm using a barometric pressure sensor was tested for the automobile which runs in real severe circumstances such as a raised expressway. The presented algorithm is also efficiently combined with the positioning algorithm that are applied by the Kalman filter and RTK-PPP method. The experimental results show that proposed method can provide continuous and accurate navigation even if there are only three visible satellites in entrance and exit of a raised expressway.

\section{References}

[1] S. Sugimoto and Y. Kubo: GNSS Regressive Models and Precise Point Positioning, Proc. of the 36th
ISCIE Int. Symp. on Stochastic Systems Theory and Its Applications, pp. 159-164, Saitama, Nov. 2004 .

[2] S. Sugimoto and Y. Kubo: GNSS Regressive Models and Precise Point Positioning, Proc. of the 36th ISCIE Int. Symp. on Stochastic Systems Theory and Its Applications, pp. 159-164, Saitama, Nov. 2004.

[3] S. Sugimoto and R. Shibasaki (Eds.): GPS Handbook, Asakura, Tokyo, 2010 (in Japanese).

[4] A. Chabata, Y. Suzuki, Y. Kubo and S. Sugimoto: RTK-PPP Algorithms using GNSS Observables from Few Satellites, Proc. of the ION GNSS 2012, pp. 3696-3707, Nashville, TN, Sept. 2012.

[5] M. Ozaki, M. Ohashi, Y. Kubo and S. Sugimoto: Improvement of GPS Precise Point Positioning Accuracy in Urban Canyons by Using Barometric Pressure Sensor. Proc. of the 45th ISCIE Int. Symo. on Stochastic Systems Theory and Its Applications, Okinawa, Nov. 2013, will appear.

[6] R. A. Singer: Estimating Optimal Tracking Filter Performance for Manned Maneuvering Targets, IEEE Trans. Aerospace and Electronic Systems, Vol. AES-6, No. 4, pp. 197-204, July, 1970.

[7] T. Aoki and S. Sugimoto: Dynamical Models for Automobile Movements, Int. J. of Innovative Computing, Information and Control, Vol. 6, No. 1, pp. 3-14, Jan., 2010.

[8] C. Uratani, K. Sone, Y. Muto, S. Maruo and S. Sugimoto: Dynamical Models for Carrier-Phase Kinematic GPS Positioning, Proc. 16th Int. Tech. Meeting of the Satellite Division of The Institute of Navigation (ION GNSS 2003), pp. 809-818, Portland, OR, Sep., 2003.

[9] Y. Muto, Y. Kubo, C. Uratani and S. Sugimoto: New Dynamical Models for Kinematic GPS Positioning, Proc. 17th Int. Tech. Meeting of the Satellite Division of The Institute of Navigation (ION GNSS 2004), pp. 2519-2528, Long Beach, CA, Sep., 2004.

[10] B. W. Parkinson and J. J. Spilker Jr. (Eds.): Global Positioning System: Theory and Applications, Vol. I, II, AIAA, Washington, DC, 1997.

[11] R. G. Brown and P. Y. C. Hwang: Introduction to Random Signals and Applied Kalman Filtering, third edition, John Wiley \& Sons, New York, 1997.

[12] T. Sakai, K. Koremura, and K. Niimi: Height Measurement Error of Barometric Altimeter and Its Correction, Electronic Navigation Research Institute Papers, No. 114, pp. 1-13, March, 2005. 